E.L. Wignall

P.D. Griffiths

N.G. Papadakis

I.D. Wilkinson

L.I. Wallis

O. Bandmann

P.E.E. Cowell

N. Hoggard

\section{ORIGINAL RESEARCH \\ Corpus Callosum Morphology and Microstructure Assessed Using Structural MR Imaging and Diffusion Tensor Imaging: Initial Findings in Adults with Neurofibromatosis Type 1}

BACKGROUND AND PURPOSE: Imaging studies have shown that children with NF-1 have increased brain volumes compared with age-matched controls and the CCs are disproportionately large. The purpose of this study was to determine if the CC in adults with NF-1 differed from that in matched controls by using DTI and volumetric imaging.

MATERIALS AND METHODS: MR imaging with DTI was performed in 10 adults with NF-1 and in 10 age-, sex-, and handedness-matched controls by using a 3T system. Total brain volumes and the areas and central lengths of the $\mathrm{CC}$ were calculated, along with the radial width of callosal subdivisions, in the 2 groups.

RESULTS: Our results showed that the total brain volume was not significantly different between adults with NF-1 and matched controls. The length and total cross-sectional area of the CC were statistically larger in adults with NF-1 compared with controls (approximately 10\% longer and 20\% greater area). On DTI we found a preservation of the primary eigenvalue with increases in the minor eigenvalues at the genu.

CoNCLUSIONS: We have shown that the increased size of the CC found in children with NF-1 is also present in adults with the syndrome, whereas no difference in total brain volume was found.

ABBREVIATIONS: $\mathrm{CC}=$ corpus callosum; $\mathrm{Cl}=$ confidence interval; $\mathrm{DTI}=$ diffusion tensor imaging; $\mathrm{FA}=$ fractional anisotropy; FLAIR $=$ fluid-attenuated inversion recovery; ICC $=$ intraclass correlation coefficients; NF-1 = neurofibromatosis type 1
$\mathbf{N}$ F-1 is the most common of the phakomatoses and affects 1 in $4000-5000$ people. It is inherited as an autosomal dominant disorder, but in $50 \%$ of people, the disorder arises as a spontaneous mutation. ${ }^{1}$ The NF-1 gene is located on chromosome 17, and it encodes for the protein neurofibromin, which is thought to act as a tumor suppressor. ${ }^{2}$ Many of the abnormalities found in NF-1 result from the "production of a nonfunctional protein or absence of its expression."3 Physical symptoms vary greatly in presentation and severity, both within the NF-1 population as a whole and within the same family. ${ }^{4}$ Children with NF-1 have a high prevalence of intracranial abnormalities with a wide range of pathology, including neoplasms (optic gliomas, $15 \%-20 \%$, and brain gliomas) ${ }^{5}$; areas of hyperintensity on $\mathrm{T} 2$ images commonly referred to as neurofibromatosis bright objects ${ }^{6,7}$; and macrocephaly, which occurs in 30\%-53\% of patients. ${ }^{8}$ Larger-than-normal brain volume (as distinct from a large calvaria) has also been found in children with NF-1 compared with healthy controls, ${ }^{3,9}$ and both gray ${ }^{6,10}$ and white matter structures are larger. ${ }^{10}$

The CC is the major commissural structure in the human

\section{Received June 16, 2009; accepted after revision October 7.}

From the Academic Unit of Radiology (E.L.W., P.D.G., N.G.P., I.D.W., L.I.W., N.H.), University of Sheffield, latropolis-Magnitiki Tomografia, Athens, Greece; and Academic Unit of Neurology (O.B.) and Department of Human Communication Sciences (P.E.E.C.), University of Sheffield, Sheffield, United Kingdom.

E.L. Wignall and P.D. Griffiths contributed equally to this work.

Please address correspondence to Nigel Hoggard, MD, Academic Unit of Radiology, University of Sheffield, Floor C, Royal Hallamshire Hospital, Glossop Rd, Sheffield S10 2JF United Kingdom; e-mail: n.hoggard@sheffield.ac.uk

DOl 10.3174/ajnr.A2005 brain. Several studies have looked at the size of the CC in children with NF-1, and all have shown increased volumes. ${ }^{3,6,11,12}$ Most of those studies corrected CC size for some assessment of total brain volume, and the increased size of the CC remained statistically significant independent of brain size. This suggests that the enlarged CC in subjects with NF-1 is proportionally greater than any increase in overall brain size. Only 3 studies have looked at the size of subdivisions in the CC in children with NF-1, ${ }^{6,11,12}$ and all found that the body of the CC in patients with NF-1 showed more volume increase than other parts.

The aim of our study was to measure the size of the CC in adults with NF-1 and to compare it with that in control subjects by using the callosal measurement methodology described by Denenberg et al. ${ }^{13,14}$ In addition, DTI was performed and may shed light on the microstructure of the CC in healthy subjects and those with NF-1.

\section{Materials and Methods}

\section{Subjects}

Ten adults with NF-1 (2 men, 8 women) were recruited through the outpatient neurology clinics based at the Royal Hallamshire Hospital, Sheffield, United Kingdom. All subjects with NF-1 had the diagnosis made in childhood by using standard clinical criteria. None of the subjects with NF-1 included in this study had a history of optic or brain glioma, none had cranial neurosurgery or radiation therapy, and their clinical condition remained stable for several years. Ten control subjects who were age-, sex-, and handedness-matched were recruited. The age range of the subjects with NF-1 was 20-68 years, 
and the range in controls was from 21 to 64 years. All subjects gave written informed consent to take part in the study with the guidance and approval of South Sheffield Ethics Committee.

\section{MR Imaging and Postprocessing}

All MR images were obtained on the same 3T superconducting system (Intera 3T; Philips Healthcare, Best, the Netherlands), and all subjects had T1-weighted whole-brain volume, DTI, and axial FLAIR imaging. The routine images were reviewed by an experienced neuroradiologist (P.D.G. or N.H.), looking specifically for unexpected pathology that would lead to exclusion from the study. The T1-weighted volume imaging consisted of a magnetization-preparation rapid acquisition of gradient echo sequence acquired in the sagittal plane and covering the entire head. Two hundred partitions of $0.8-\mathrm{mm}$ thickness and $0.8 \times 0.8 \mathrm{~mm}$ in-plane resolution were obtained, producing high-resolution anisotropic data (TR, $11 \mathrm{~ms}$; TE, $4.8 \mathrm{~ms}$; acquisition matrix, $256 \times 256$; FOV, $204.8 \mathrm{~mm}$; 12-minute acquisition time). Each dataset was then oriented in the sagittal plane, and the hemispheres were separated. This was to ensure a clear midline section and reduce any unnecessary postprocessing by having to reorient the baseline in accordance with previously described volume postprocessing procedures. ${ }^{15}$

DTI data were acquired by using a single-shot diffusion-weighted spin-echo-planar sequence described by Stejskal and Tanner. ${ }^{16}$

The diffusion-weighted acquisitions $\left(b=1000 \mathrm{~s} / \mathrm{mm}^{2}\right)$ were from 16 distinct diffusion-weighted gradient directions with a single baseline non-diffusion weighted $b=0 \mathrm{~s} / \mathrm{mm}^{2}$. Fifty contiguous axial sections covering the whole of the supratentorial compartment were acquired by using a sensitivity encoding factor of $1.75(\mathrm{TR} \approx 7550 \mathrm{~ms}$; TE, $84 \mathrm{~ms}$; acquisition matrix, $128 \times 128$; section thickness, $2 \mathrm{~mm}$; FOV, $224 \mathrm{~mm}$; acquired in 6 minutes).

Sixteen-bit raw image files were created for each subject's 3D whole-brain volume by using proprietary scanner software and were converted to Analyze format by using MRIcro (http://www.sph.sc. edu/comd/rorden/mricro.html). Gray and white matter volumes, including the brain stem and cerebellum, were calculated by quantifying gray and white matter "concentrations" from the segmented maps produced in SPM2 (http://www.fil.ion.ucl.ac.uk/spm). This was achieved by multiplying the number of voxels classified as gray or white matter with known voxel size to produce a volume estimate (script taken from http://www.sph.umich.edu/ nichols/JohnsGems. html\#Gem15). The resulting gray and white matter volumes were added together to give an estimate of whole-brain volume, including the brain stem and cerebellum.

All measurements of the CC were made on a 2D midsagittal section. To avoid the effect that intersubject variation in head orientation may have on section selection, we applied a standardized brain orientation, following the methodology described by Howard et al. ${ }^{15}$ The midsagittal image was defined by visualization of the following landmarks: 1) the cerebellum and brain stem clearly separated by the 4 th ventricle, 2) fluid space separating the tectum and tegmentum, 3) the maximal area of the cerebellar vermis, 4) minimal cortical gray matter, and 5) fluid space separating the CC and cingulate gyrus. The linear craniocaudal length and total cross-sectional area of the CC were measured by 2 researchers (E.L.W. and L.I.W.). The outline of the CC was then traced manually and divided into percentiles by 99 equally spaced lines perpendicular to the long axis of the CC. The software for this task was developed locally (P.E.E.C.) but was based on the principles described by Denenberg et al. ${ }^{13}$ This allows regional estimates of the CC width. The 99 width measurements were then

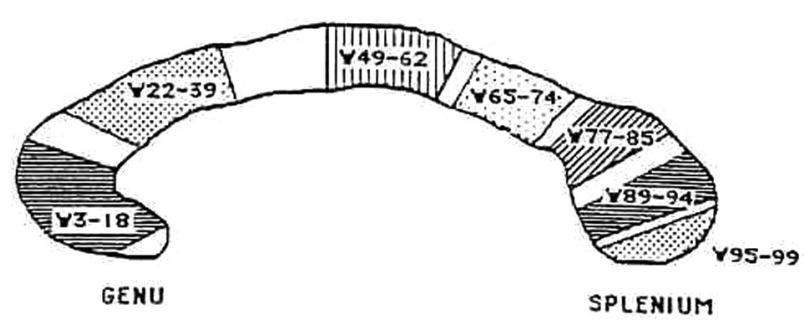

Fig 1. Line diagram of the divisions of the CC used for regional width measurements and factor structure by using the method of Denenberg et al $(1991)^{13}$ and Denenberg et al (1991). ${ }^{14}$ See text for details.

grouped into clusters following the factor analytic approach of the original work of Denenberg et $\mathrm{al}^{14}$ : 3-18 (F1), 22-39 (F2), 49-62 (F3), 65-74 (F4), 77-85 (F5), 89-94 (F6), and 95-99 (F7) as shown in Fig 1 . The stability of the factor structure has been replicated within and between laboratories. ${ }^{17,18}$

Sixteen-bit raw image files were created for each DTI dataset and corrected for eddy current artifacts and subject motion by using a proprietary motion-correction tool (PRIDE, Philips Healthcare). The software uses affine transformations to register the images, which preserves both colinearity (points in a straight line remain in a straight line) and distance ratios. In-house $\mathrm{C}$ programs were used to fit corrected data to the diffusion tensor equation, to produce FA, all 3 eigenvalues, and trace data maps in Analyze format. Regions of interest were placed on the FA maps in the posterior body/isthmus region of the CC (approximating F4) and in the genu of the CC (approximating F1) by using MEDX (Sensor Systems, Sterling, Virginia). Regions of interest were rectangular, consisting of 15 pixels $(5 \times 3)$ in $\mathrm{F} 1$ and 20 pixels $(5 \times 4)$ in F4. The FA value, trace value, and 3 eigenvectors were calculated, and the 2 smallest eigenvalues were averaged to produce a composite of radial diffusion $\left(\lambda_{\perp}=\left(\lambda_{2}+\lambda_{3}\right) / 2\right)$. Averaging is considered advisable to reduce noise that can be high in the single smaller eigenvalues. ${ }^{19}$

\section{Statistical Methods}

The Kolomogorov-Smirnov test was used to check that the data conformed to a normal distribution, and subsequently unpaired 2-tailed $t$ tests were used for comparing both volumetric and diffusion parameters. A simple Bonferroni correction $(P<.05 /$ number of comparisons) was applied to correct for multiple comparisons for both volumetric and diffusion comparisons, but $P$ values of $<.05$ and $<.01$ before correction were also reported. Both inter- and intrarater reliability of CC measurements was assessed by using ICC (2-way mixed design) with 8 images for both the callosum measures and region-ofinterest placement. The ICC was calculated for all the diffusion parameters of the 2 regions of interest due to the difference in the signal intensity-to-noise characteristics of the different diffusion parameter maps.

\section{Results}

All MR imaging examinations produced high-quality data, there were no unexpected imaging findings, and none of the subjects had any neurofibromatosis bright objects at the time of the examination, though clearly it is unknown whether they had had any as children. The Kolomogorov-Smirnov analysis of the volume data showed that the distributions of the measurements did not differ significantly from a normal distribution $(P>.10)$, justifying the use of parametric statistics. There was no significant difference in brain volumes of subjects with 


\begin{tabular}{|c|c|c|c|}
\hline & $\begin{array}{l}\text { Patients with NF-1 } \\
\quad(n=10)\end{array}$ & $\begin{array}{l}\text { Matched Controls } \\
(n=10)\end{array}$ & $\begin{array}{c}t \text { Statistic } \\
(d f=9)\end{array}$ \\
\hline \multicolumn{4}{|l|}{ Global CC measures } \\
\hline Central length (mm) & $94.1(5.7)$ & $85.4(5.7)$ & $3.46^{b}$ \\
\hline Area $\left(\mathrm{mm}^{2}\right)$ & $708.4(74.9)$ & $587.2(53.8)$ & $4.15^{b}$ \\
\hline Total brain volume $\left(\mathrm{cm}^{3}\right)$ & $1050(110)$ & $1010(90)$ & 0.89 \\
\hline \multicolumn{4}{|l|}{ Factor groupings $(\mathrm{mm})$} \\
\hline F1 (widths 3-18) & $13.0(3.2)$ & $14.5(1.6)$ & 1.33 \\
\hline F2 (widths 22-39) & $9.2(1.7)$ & $7.3(0.7)$ & $3.21^{b}$ \\
\hline F3 (widths 49-62) & $7.9(1.4)$ & $6.6(0.7)$ & $2.58^{\mathrm{c}}$ \\
\hline F4 (widths 65-74) & $7.9(0.8)$ & $6.2(0.7)$ & $5.09^{b}$ \\
\hline F5 (widths 77-85) & $12.7(1.9)$ & $10.8(1.2)$ & $2.67^{\mathrm{C}}$ \\
\hline F6 (widths 89-94) & $14.3(1.7)$ & $13.2(1.9)$ & 1.40 \\
\hline F7 (widths 95-99) & $5.1(0.4)$ & $4.6(0.3)$ & $2.55^{\mathrm{c}}$ \\
\hline
\end{tabular}

a There is no significant difference between the total brain volumes of the subjects with NF-1 and controls, but the area and length of the CC are larger in NF-1. The factor groupings are also presented pictorially in Fig 1.

b $P<.05$ after the Bonferroni correction.

${ }^{c} P<.05$ before the Bonferroni correction (.05/10) only.

NF-1 and the age-matched control group $(P>.10)$. All ICC for CC measures (both consistency and absolute agreement) were $>0.85$, demonstrating good reliability and repeatability between measures.

Table 1 presents the information concerning the size of the $\mathrm{CC}$ from the 2 groups. The overall area of the $\mathrm{CC}$ was larger in subjects with NF-1 compared with controls (by approximately $20 \%$ ), and this reached statistical significance both before and after the Bonferroni correction $\left(t_{18}=4.15, P<.001\right)$. The length of the CC was larger in people with NF-1 compared with controls (by approximately $10 \%$ ), which reached statistical significance before and after the Bonferroni correction.

All regional subdivisions of $\mathrm{CC}$ width tended to be larger in individuals with NF-1 except in F1 (the most rostral region of the CC) as shown in Fig 2. Factor width groupings F2 and F4 (anterior and posterior body) were significantly wider before and after a Bonferroni correction (0.05/10). In many cases, the increased size of the CC was obvious on visual inspection of the images.

DTI data are presented in Table 2. ICC measures for both the anterior and posterior region-of-interest placement produced high levels of agreement $(>0.93$ in all cases). FA was significantly reduced in subjects with NF-1 compared with controls in both the genu and the posterior body region of interest before and after Bonferroni correction $(P<.05 / 8)$ (Fig 3). There was no statistically significant difference in the primary eigenvalues (longitudinal diffusion, $\lambda_{\|}$) in either the genu or the posterior body. The combined 2 smaller eigenvalues, "radial diffusion" $\left(\lambda_{\perp}\right)$, were significantly larger in the NF-1 group at both sites before the Bonferroni correction and remained so after correction in the genu $\left(t_{18}=4.459, P=\right.$ $.003)$. There was no difference in trace in the genu or posterior body of the CC before or after Bonferroni correction $(P>$ $.10)$.

\section{Discussion}

Macrocephaly is a well-recognized feature in children with NF-1. This has led many groups to look for parts of the brain that may be specifically involved. Said et $\mathrm{al}^{10}$ found larger gray matter volumes in children with NF-1, but the magnitude was small $(<10 \%)$, though greater in the boys with NF-1 than in the girls with NF-1 compared with controls. In contrast, the magnitude of increased white matter volume was much larger in NF-1 (>18\% compared with age-matched controls), and this feature was most apparent in girls compared with controls ( $>45 \%$ white matter volume compared with age-matched controls). Moore et $\mathrm{al}^{6}$ found that the differences in gray-towhite-matter ratios seen in subjects with NF-1 tended to reduce with age.

The CC is an ideal substrate for assessment with DTI: It is relatively large, has a very predictable microstructure, and is relatively easy to outline and measure anatomically compared with the deep white matter regions of interest, for example, reported by previous groups in NF-1. ${ }^{20,21}$ There is broad correlation between the area of the $\mathrm{CC}$ and total brain volume, though the relationship between regional assessment of CC size and whole-brain volume in controls is not clearly established. It appears to depend on the technique of assessing the whole-brain size (wet weight, midsagittal area, forebrain volume) and the method of CC measurement used. ${ }^{22}$

If a population has larger whole brain volumes, it is essential to consider that differences in CC size might be purely a consequence of larger brain volumes. Moreover, the CC continues to grow throughout childhood and early adulthood, ${ }^{23-25}$ but the growth is unequal, with some regions growing at different rates than others. ${ }^{26,27}$

One possible explanation for the increased size of the CC in children with NF-1 is a delay and/or reduction in apoptosis, 3,6 which would produce greater numbers of commissural fibers. Other possible causes include larger axons due to excessive myelination, increased extracellular fluid, myelinopathy associated with vacuolation, ${ }^{20,28}$ or a combination of those factors. Moore et $\mathrm{al}^{6}$ found that differences in gray matter volume were more pronounced in younger subjects with NF-1, which led them to suggest that apoptosis was delayed rather than absent. If that is the case, it is predicted that there would be little or no difference in the size of the CC between adults with NF-1 and age-matched controls. We have shown that the CC in adults (age range, 20-68 years) with NF-1 is larger than that in matched controls and that the degree of difference is comparable with the findings in children with NF-1. , $^{3,6,11,12}$ We have also shown that this finding in our group of patients with NF-1 is not related to overall differences in brain volume. The larger 


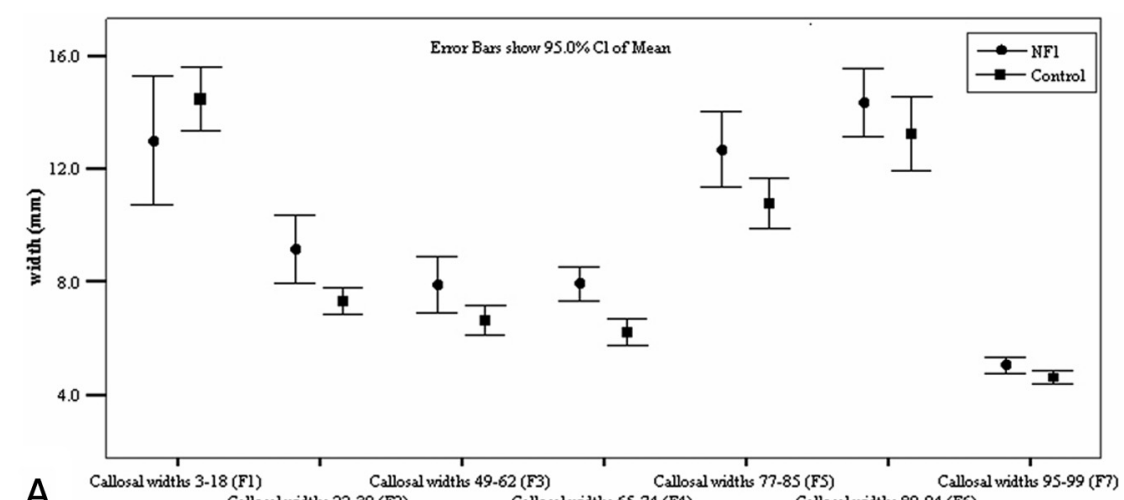

A

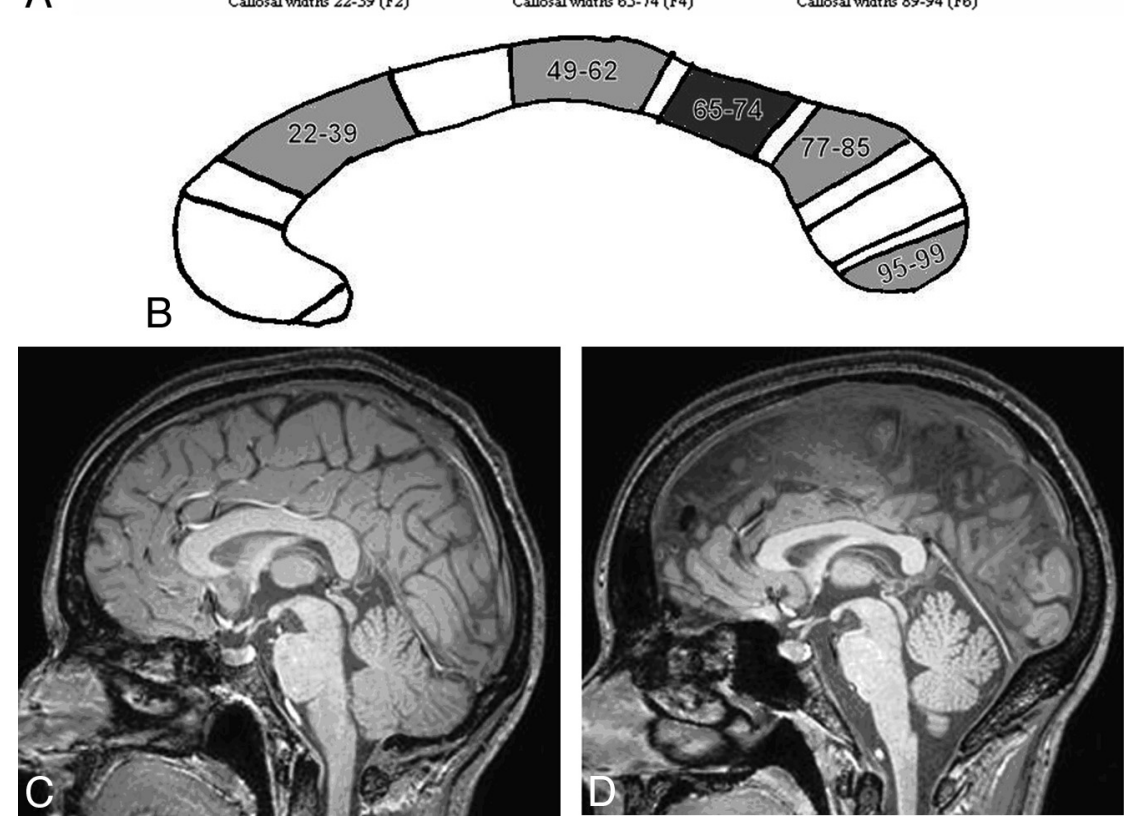

Fig 2. $A$, The mean widths ( $\pm 95 \% \mathrm{Cls}$ ) of the individual factors for both subjects with NF-1 and controls. $B$, Schematic representation of the regions of the CC that are statistically significantly wider in subjects with NF-1 compared with controls (white indicates not significant; light gray, significant to $P<.05$; and dark gray, significant to $P<.005$ ). $C$ and $D$, Midsagittal images from the T1-weighted volume dataset from a person with NF-1 (C) and a matched control (D). The CC is visibly larger in the person with NF-1, particularly in the posterior aspect.

brain volume and CC size in children with NF-1 have been attributed to reduced/delayed apoptosis. ${ }^{3,6}$

Apoptosis occurs in many species and across many aspects of nervous system development and maturation. ${ }^{29}$ For example, the Rhesus monkey loses $70 \%$ of its callosal axons after

\begin{tabular}{|c|c|c|c|}
\hline & $\begin{array}{l}\text { Patients with NF-1 } \\
\quad(n=10)\end{array}$ & $\begin{array}{l}\text { Matched Controls } \\
(n=10)\end{array}$ & $\begin{array}{l}t \text { Statistic } \\
(d f=18)\end{array}$ \\
\hline \multicolumn{4}{|l|}{$\overline{\text { Genu }}$} \\
\hline FA & $0.68(0.06)$ & $0.77(0.05)$ & $3.644^{b}$ \\
\hline$\lambda_{\|}$ & $1.92(0.35)$ & $1.91(0.14)$ & 0.084 \\
\hline$\lambda_{\perp}{ }^{\mathrm{c}}$ & $0.52(0.07)$ & $0.39(0.06)$ & $4.46^{\mathrm{b}}$ \\
\hline Trace & $0.99(0.16)$ & $0.89(0.04)$ & 0.36 \\
\hline \multicolumn{4}{|l|}{ Body } \\
\hline FA & $0.70(0.05)$ & $0.77(0.04)$ & $3.46^{b}$ \\
\hline$\lambda_{\|}$ & $1.66(0.08)$ & $1.77(0.13)$ & $2.28^{d}$ \\
\hline$\lambda_{\perp}{ }^{\mathrm{c}}$ & $0.42(0.07)$ & $0.35(0.06)$ & $2.40^{\mathrm{d}}$ \\
\hline Trace & $0.83(0.06)$ & $0.82(0.06)$ & 0.37 \\
\hline
\end{tabular}

a See text for discussion.

b $P<.05$ after Bonferroni correction.

${ }^{\mathrm{c}}$ Diffusion trace, $\lambda_{\|}$, and $\lambda$, are expressed in $10^{-3} \mathrm{~mm}^{2} / \mathrm{s}$.

$\mathrm{d} P<.05$ before Bonferroni correction only (.05/8). birth ${ }^{26}$ though the adult pattern of callosal connections is present at least 1 month before birth. ${ }^{30,31}$ As the CC grows postnatally, transitory axons are lost through apoptosis, while the remaining axons increase in diameter and myelinate, decreasing the attenuation of axons in a given callosal area. ${ }^{30}$

Assuming that a comparable process of apoptosis occurs in humans as has been show in Rhesus monkeys, one could reason a priori that failed or delayed apoptosis would produce larger total gray matter volumes, larger total white matter volumes, and larger CC size in NF-1. Those are the exact findings of neuroimaging studies in children with NF-1 reported in the literature. ${ }^{3,6,11,12}$ If apoptosis is delayed in NF-1, as opposed to failed, the increased volumes found in children with NF-1 should normalize with time. It is likely that the ages of our adult group were sufficiently high (range, 20-68 years) to assume that any modifications that were going to occur had already happened and that no further myelination would occur. The finding of equal total gray and white matter volume in our 2 groups supports the theory of delayed apoptosis rather than failed apoptosis because of the normalization of brain volumes in adults 

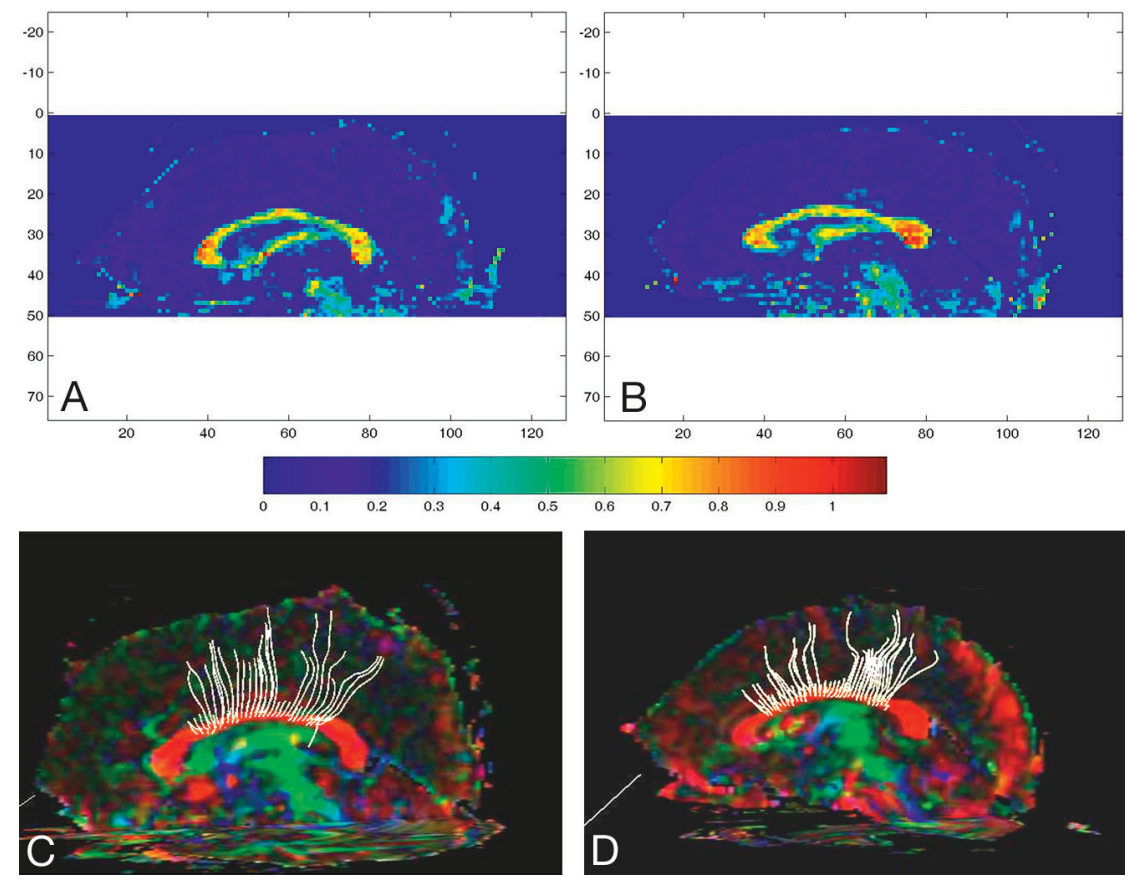

Fig 3. $A$ and $B$, Sagittally reformatted color FA maps for a subject with NF-1 $(A)$ and a matched control $(B)$, with color-scale guide below. $C$ and $D$, Tractography for the subject with NF-1 $(C)$ and the control $(D)$.

with NF-1, though this needs confirmation in a larger study.

The observation that the CC remains enlarged in adults with NF-1 requires further discussion because this appears different from the response of other parts of the brain. The posterior body and isthmus of the CC were shown to be the most consistently enlarged portions in adults with NF-1, though virtually all regions showed some increase in width. Previous studies of children with NF-1 have reported the greatest changes in the more anterior regions of the body of the CC. ${ }^{6,11,12}$

We have attempted to uncover the cause of the persisting increased size of the CC in NF-1 by performing DTI studies. This is an MR imaging method that uses water diffusion to provide clues to the microstructure of the brain in vivo. ${ }^{32}$ The presence of axons and their associated myelin imposes directionality to the diffusion of water. The magnitude of the favored and nonfavored diffusion characteristics can be calculated as eigenvectors/eigenvalues. Diffusion along the length of the axon is the favored longitudinal or primary eigenvalue $\left(\lambda_{\|}\right)$, with 2 smaller transverse eigenvalues that have been averaged in this study to produce a measure of "radial diffusion" $\left(\lambda_{\perp}\right)$.

Our results showed diffusion changes in the CC of subjects with NF-1 compared with controls, though the differences were relatively small. There was statistically significant reduced FA with increased radial diffusivity at both sites of the CC studied, but this remained significant after Bonferroni correction only for radial diffusivity in the genu of the CC. The reduced FA (with normal trace) was predominantly the result of increased radial diffusion in subjects with NF-1.

In the 2 previous reports of DTI in NF-1, ${ }^{20,21}$ white matter changes were found that affected both normal- and ab- normal-appearing white matter. The first, van Engelen et $\mathrm{al}^{20}{ }^{20}$ did not report the findings of the eigenvalues; the latter, Zamboni et al, ${ }^{21}$ did, reporting falls in all $\lambda_{1}, \lambda_{2}$, and $\lambda_{3}$. It has been postulated in the Zamboni study of pediatric patients with NF-1 on the basis of all 3 eigenvalues being reduced that the primary cause for the reduced FA was related to vacuolation or edema of the myelin, as has been found on pathologic study of the brain. ${ }^{10,33}$ However in our study, we have found a preservation of the primary eigenvalue with increases in the minor eigenvalues. The lack of increase in the primary eigenvalue argues against myelin vacuolation as an explanation, given that this random process should affect the diffusion tensor in all directions. These DTI findings will be the subject of further study, with a larger population that will allow age as a factor to be evaluated. Also a less selected group of patients with NF-1 is now required, though the presence of coexisting pathology may, in turn, complicate interpretation.

In summary, our findings in this small group show that the gray and white matter volumes of adults with NF-1 do not differ statistically from those in controls, unlike children with NF-1 who have larger gray and white matter volumes. In contrast, the CC in the adult-with-NF-1 population is larger than that in controls, a finding that mirrors the situation in children with NF-1. Future larger studies are needed to investigate the growth of the callosum and accompanying diffusion characteristics longitudinally in children with NF-1 to establish whether the regions of the CC found to be larger in size in the NF-1 group in the current study are due to subject or methodologic differences. It would also be of interest to begin to study differences in CC morphology compared with the neuropsychological differences that have been found in pediatric patients with NF-1, because diffusion results may give a more complete picture 
of functional integrity than volumetric/morphometric measurements alone.

\section{Acknowledgments}

We acknowledge the skill and hard work of the MR imaging radiographers of the Academic Unit of Radiology, University of Sheffield.

\section{References}

1. Huson SM, Harper PS, Compston DA. Von Recklinghausen neurofibromatosis: a clinical and population study in south-east Wales. Brain 1988; 111(pt 6):1355-81

2. Bennett MR, Rizvi TA, Karyala, et al. Aberrant growth and differentiation of oligodendrocyte progenitors in neurofibromatosis type 1 mutants. J Neurosci 2003;23:7207-17

3. Dubovsky EC, Booth TN, Vezina G, et al. MR imaging of the corpus callosum in pediatric patients with neurofibromatosis type 1 . AJNR Am J Neuroradiol 2001;22:190-95

4. Huson SM. Neurofibromatosis 1: a clinical and genetic overview. In: Huson SM, Hughes RAC, eds. The Neurofibromatoses: A Pathogenetic and Clinical Overview. London: Chapman \& Hall Medical; 1994;160-203

5. Listernick R, Charrow J, Greenwald MJ, et al. Optic gliomas in children with neurofibromatosis type 1. J Pediatr 1989;114:788-92

6. Moore BD 3rd, Slopis JM, Jackson EF, et al. Brain volume in children with neurofibromatosis type 1: relation to neuropsychological status. Neurology 2000;54:914-20

7. Griffiths PD, Mukunoveshuro W, Blaser S, et al. The natural history of T2 hyperintensities in children with neurofibromatosis type 1: a proliferative potential. Pediatrics 1999;104:e49

8. Bale SJ, Amos CI, Parry DM, et al. Relationship between head circumference and height in normal adults and in the nevoid basal cell carcinoma syndrome and neurofibromatosis type I. Am J Med Genet 1991;40:206-10

9. Cutting LE, Koth CW, Burnette CP, et al. Relationship of cognitive functioning, whole brain volumes, and T2-weighted hyperintensities in neurofibromatosis-1. J Child Neurol 2000;15:157-60

10. Said SM, Yeh TL, Greenwood RS, et al. MRI morphometric analysis and neuropsychological function in patients with neurofibromatosis. Neuroreport 1996;7:1941-44

11. Mott SH, Baumgartner T, Vezina G, et al. Neurofibromatosis type 1: corpus callosum enlarged beyond megacephaly. Ann Neurol 1996;40:325

12. Kayl AE, Moore BD 3rd, Slopis JM, et al. Quantitative morphology of the corpus callosum in children with neurofibromatosis and attention-deficit hyperactivity disorder. J Child Neurol 2000;15:90-96

13. Denenberg VH, Cowell PE, Fitch RH, et al. Corpus callosum: multiple parameter measurements in rodents and humans. Physiol Behav 1991;49:433-37

14. Denenberg VH, Kertesz A, Cowell PE. A factor analysis of the human's corpus callosum. Brain Res 1991;548:126-32

15. Howard MA, Roberts N, Garcia-Finana M, et al. Volume estimation of prefron- tal cortical subfields using MRI and stereology. Brain Res Brain Res Protoc 2003;10:125-38

16. Stejskal EO, Tanner JE. Spin diffusion measurements: spin echoes in the presence of time-dependent field gradients. J Chem Phys 1965;42:288-92

17. Cowell PE, Allen LS, Kertesz A, et al. Human corpus callosum: a stable mathematical model of regional neuroanatomy. Brain Cogn 1994;25:52-66

18. Peters M, Oeltze S, Seminowicz D, et al. Division of the corpus callosum into subregions. Brain Cogn 2002;50:62-72

19. Martin KM, Papadakis NG, Huang CL, et al. The reduction of the sorting bias in the eigenvalues of the diffusion tensor. Magn Reson Imaging 1999;17:893-901

20. van Engelen SJ, Krab LC, Moll HA, et al. Quantitative differentiation between healthy and disordered brain matter in patients with neurofibromatosis type I using diffusion tensor imaging. AJNR Am J Neuroradiol. 2008;29:816-22

21. Zamboni SL, Loenneker T, Boltshauser E, et al. Contribution of diffusion tensor MR imaging in detecting cerebral microstructural changes in adults with neurofibromatosis type 1. AJNR Am J Neuroradiol 2007;28:773-76

22. Jäncke L, Steinmetz H. Brain size: a possible source of interindividual variability in corpus callosum morphology-the parallel brain: the cognitive neuroscience of the corpus callosum. In: E. Zaidel E, M. Iacoboni M, eds. Issues in Clinical and Cognitive Neuropsychology. Cambridge, Massachusetts: MIT Press; 2003:51-63

23. Pujol J, Vendrell P, Junque C, et al. When does human brain development end? Evidence of corpus callosum growth up to adulthood. Ann Neurol 1993;34: $71-75$

24. Weis S, Kimbacher M, Wenger E, et al. Morphometric analysis of the corpus callosum using MR: correlation of measurements with aging in healthy individuals. AJNR Am J Neuroradiol 1993;14:637-45

25. Keshavan MS, Diwadkar VA, DeBellis M, et al. Development of the corpus callosum in childhood, adolescence and early adulthood. Life Sci 2002;70: 1909-22

26. Cowell PE, Allen LS, Zalatimo NS, et al. A developmental study of sex and age interactions in the human corpus callosum. Brain Res Dev Brain Res 1992;66: $187-92$

27. Giedd JN, Rumsey JM, Castellanos FX, et al. A quantitative MRI study of the corpus callosum in children and adolescents. Brain Res Dev Brain Res 1996;91: $274-80$

28. Di Paolo DP, Zimmerman RA, Rorke LB, et al. Neurofibromatosis type I: pathologic substrate of high-signal-intensity foci in the brain. Radiology 1995; 195:721-24

29. Barinaga M. Death gives birth to the nervous system. But how? Science 1993;259:762-63

30. LaMantia AS, Rakic P. Axon overproduction and elimination in the corpus callosum of the developing rhesus monkey. J Neurosci 1990;10:2156-75

31. Killackey HP, Chalupa LM. Ontogenetic change in the distribution of callosal projection neurons in the postcentral gyrus of the fetal rhesus monkey. J Comp Neurol 1986;244:331-48

32. Beaulieu $C$. The basis of anisotropic water diffusion in the nervous system: a technical review. NMR Biomed 2002;15:435-55

33. Sheikh SF, Kubal WS, Anderson AW, et a;. Longitudinal evaluation of apparent diffusion coefficient in children with neurofibromatosis type 1. J Comput Assist Tomogr 2003;27:681-86 